\title{
Effect of bio-loading on polyvinyl chlorides morphology
}

\section{Effet du bio-chargement sur la morphologie des polychlorures de vinyle}

\author{
M.JAMMOUKH ${ }^{1}$, K.MANSOURI ${ }^{1}$ et B.SALHI ${ }^{2}$ \\ ${ }^{1}$ University of Hassan II, Laboratory of Signals, Distributed Systems and Artificial Intelligence, Casablanca, Morocco \\ ${ }^{2}$ University of Mohammed V, Normal Superior School of Technical Education, Rabat, Morocco.
}

\begin{abstract}
Résumé Permettant d'intégrer la prise en compte des questions environnementales lors de la phase de conception d'un produit, l'éco-conception est une démarche préventive d'amélioration de la qualité écologique de celui-ci tout en vérifiant que le gain environnemental obtenu ne se fait pas au détriment d'autres aspects environnementaux et se focalisant sur la conservation de sa qualité d'usage de ses caractéristiques ou de certaines étapes de son cycle de vie sans faire perdre pour autant la vision globale propre à l'éco-conception. Suite à cette vision, l'utilisation des polymères bio-chargés à base animale, issue d'un mode de production mettant en œuvre des pratiques d'optimisation respectueuses des ressources naturelles de manière durable, s'impose comme changement de manière de penser à remplacer largement l'usage de ressources non renouvelables pour les activités humaines, l'épuisement des gisements de ressources fossiles et à lutter contre l'impact sur le changement climatique généré par cette pratique. Notre contribution tente d'analyser auparavant le comportement mécanique d'une bio-charge et ensuite l'incorporer dans un matériau polymère pour tester l'amélioration des caractéristiques mécaniques et thermiques apportées à ce dernier après addition et optimiser, par la suite, son emploi. C'est une charge, sous forme de poudre ou coupeaux extraits à partir d'une gaine animale. Des essais de traction sont effectués, dans un premier temps, en vue de caractériser mécaniquement le matériau vierge et ensuite le nouveau éco-composite obtenu après l'alliance, à savoir la contrainte maximale à la rupture, la déformation relative et le module de l'élasticité, ... etc.
\end{abstract}

Mots clés: Eco-conception, Polymères bio-chargés, Bio-charge, Eco-composites.

\begin{abstract}
To integrate the consideration of environmental issues in the design phase of a product eco-design is an ecological quality improvement and preventive approach of it while verifying that the resulting environmental gain is not at the expense of other environmental aspects and focusing on the preservation of its quality of use of its features or certain stages of its life cycle without losing the own global vision of eco-design . Following this vision, the use of bio-laden animal based polymers derived from a mode of production by implementing optimization practices respectful of natural resources sustainably, has become changing way of thinking largely replace the use of non-renewable resources for human activities, the depletion of fossil resources deposits and to fight against the impact of climate change generated by this practice. Our paper attempts to analyze first the mechanical behavior of a bio-load and then incorporated into a polymer material to test improved mechanical and thermal characteristics made to it after adding and optimizing subsequently, its use. This is a load in the form of powder or chips extracted from an animal sheath. Tensile tests are performed in a first step, in order to mechanically characterize the virgin material and eco-composite obtained after the combination, i.e. the maximum tensile strength, the relative deformation and the modulus of the elasticity, etc
\end{abstract}




\section{Introduction}

Le choix d'une matière repose, en effet, sur trois principales motivations pour un industriel :

- Optimiser ses coûts et varier son approvisionnement afin d'être toujours compétitif en cas de fluctuation des marchés,

- Obtenir des nouvelles fonctionnalités et propriétés,

- Réduire son empreinte environnementale [1].

Sachant qu'un produit biosourcé ne signifie pas qu'il soit à $100 \%$ dérivé de la biomasse, il est fréquent d'avoir des produits mixant plusieurs origines (fossile, minérale, végétale ou encore animale), afin de bénéficier des avantages de chaque constituant [2-7].

Parmi les matériaux basiques choisis pour la caractérisation en perspective, le Polychlorure de vinyle (PVC) souple ou rigide.

Principaux avantages du PVC: Très économique, bonne résistance chimique, intrinsèquement ignifugé.

- PVC souple : Flexible, transparent.

-PVC rigide : Bonne résistance mécaniques, transparence, bonne résistance aux UV [3].

Principaux Inconvénients du PVC : Transformation délicate et peut provoquer une dégradation rapide du matériau, résistance chimique limitée aux solvants, décoloration lors de fortes expositions aux UV, résistance en température limitée [3].

\section{Procédure expérimental de l'éco- matériau}

Le développement d'un nouveau polymère, sa synthèse à l'échelle industrielle et la mise au point des procédés de sa transformation adéquates sont des étapes longues et difficiles [4]. La voie consiste à mélanger ou à associer des polymères connus pour en combiner les propriétés, à l'instar des métallurgistes qui obtiennent des alliages dotés de propriétés nouvelles en mélangeant différents métaux. Dans une application industrielle, c'est effectivement la compatibilité qui sera un critère déterminant, puisqu'on cherchera à mettre en adéquation les propriétés d'un matériau -éventuellement multiphaséet un cahier des charges, sans forcément se soucier de la morphologie [5]. Quand deux polymères de natures différentes sont miscibles, on obtient un mélange homogène qui se comporte comme un seul polymère dont les caractéristiques dépendent de celles des polymères « Parents ».

Les véritables mélanges, ceux qui conduisent à des matériaux homogènes, sont des cas d'espèce. Souvent, les deux polymères ne sont pas miscibles (on parlera quand même encore de mélange) et au niveau microscopique, on distingue plusieurs phases [6].

\subsection{Elaboration des éprouvettes}

\subsubsection{Procédé des mélanges}

Dans le cas des mélanges, en plus des aspects thermodynamiques et chimiques, il est nécessaire de considérer les aspects mécaniques de la mise en œuvre de mélanges de polymères [7].

Pour des raisons à la fois techniques et économiques, un mélange en solution n'est guère à envisager dans le cadre d'un procédé industriel. Le procédé d'extrusion est bien adapté pour mélanger en continu des matériaux visqueux tels que les polymères [8].

\subsubsection{Procédé d'extrusion}

En général, les mélanges sont souvent mis en forme à l'état fondu dans des machines plus ou moins standard du type extrudeuses dont le profil de vis devra être choisi avec soin pour "optimiser" la dispersion et la distribution d'un polymère dans l'autre.

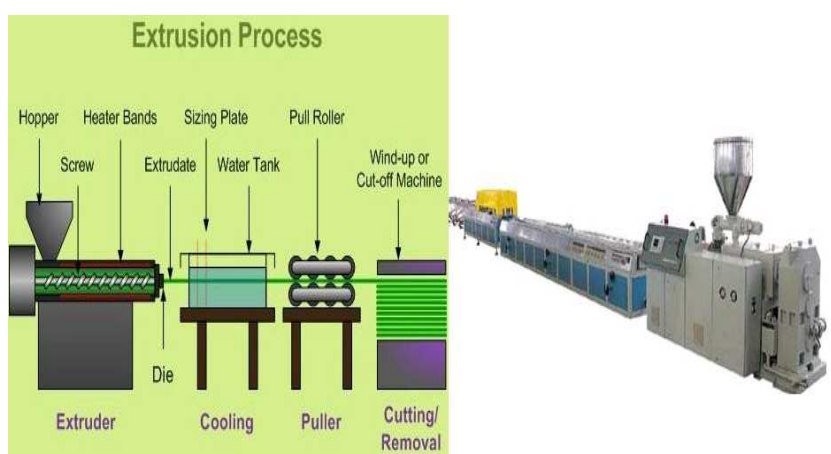

Fig.1. Procédé d'extrusion

Concernant les principes régissant le mécanisme de mélange de deux liquides visqueux non miscibles tels que les polymères, on distingue souvent le mélange dispersif et le mélange distributif dont les caractéristiques sont données ci-après :

\begin{tabular}{|l|l|}
\hline \multicolumn{1}{|c|}{ Mélange dispersif } & \multicolumn{1}{|c|}{ Mélange distributif } \\
\hline -Réduit la taille de la phase & $\begin{array}{l}\text {-Répartit de façon } \\
\text { homogène la phase } \\
\text { dispersée dans la matrice }\end{array}$ \\
-Fortes contraintes de & \\
cisaillement. & \\
-Forte viscosité. & \\
\hline
\end{tabular}




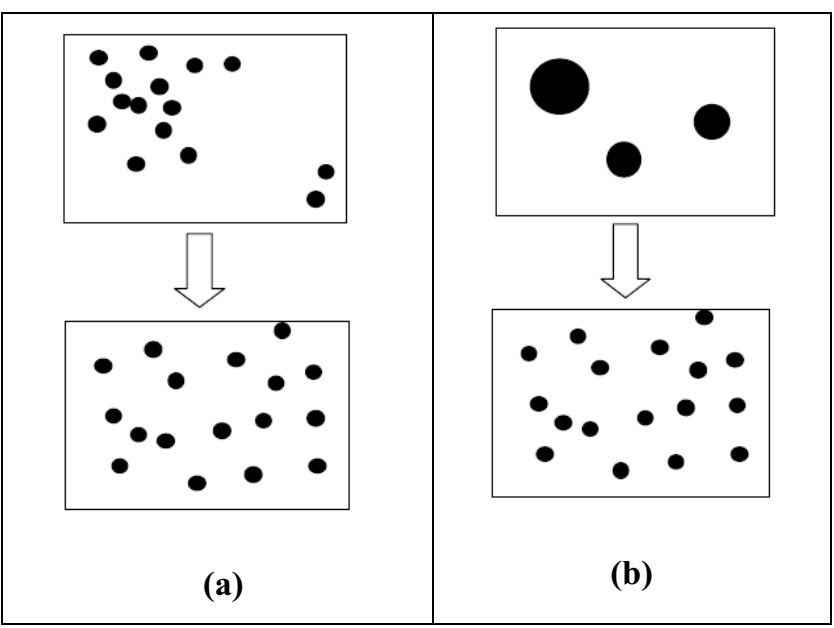

Fig.2. Mélange visqueux non miscible

Les mélanges de polymères compatibles sont rares, toute fois le PVC, par la présence de son hydrogène en $\alpha$, est miscible avec un grand nombre de polymères [5].

Les mélanges de polymères à base de PVC ont pour but :

- De faciliter la mise en œuvre du PVC lui même, en diminuant à la fois sa température de transition vitreuse (Tg) et sa viscosité.

- D'améliorer sa résistance aux chocs puisque le PVC présente une fragilité importante s'il n'est pas renforcé.

L'utilisation des matériaux conduit à définir les propriétés qualitatives que l'on attend d'eux. Chacune de ces qualités correspond respectivement à une propriété mécanique bien déterminée :

- La ténacité : Résistance à l'extension ou à la compression.

- La résilience : Résistance aux chocs.

- La dureté : Résistance à l'usure.

\subsubsection{Formage des éprouvettes de traction}

Cette étape permet d'obtenir des éprouvettes pour les essais mécaniques par le procédé de poinçonnage, particulièrement ceux des essais de traction en lots de cinq éprouvettes conformes à la norme en vigueur ISO $527-2$, type $1 \mathrm{BA}$ et de forme « Haltère ».

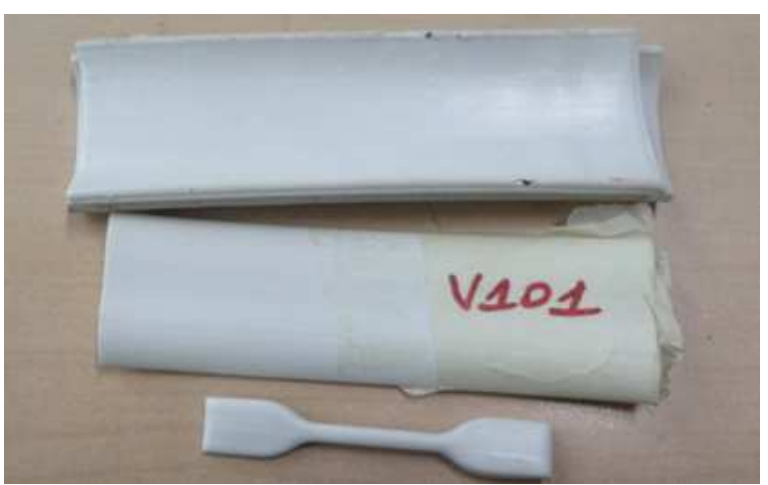

Fig.3. Profilés plats \& éprouvette type 1BA
\& de forme « Haltère » du PVC rigide vierge

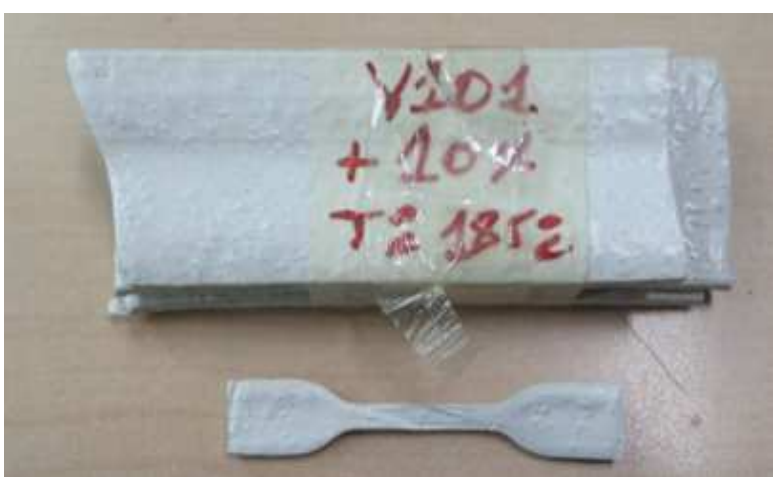

Fig.4. Profilés plats \& éprouvette type 1BA $\&$ de forme « Haltère » du PVC rigide bio-chargé

\subsection{Mode expérimental}

Pour mesurer ces propriétés mécaniques, il est nécessaire d'effectuer des essais. Ces derniers ont pour objet de tester le comportement d'un matériau dans des conditions conventionnelles voisines des situations pratiques [9].

\subsubsection{Machine utilisée}

Pour l'essai de traction, une machine de tractioncompression universelle de type LLOYD Instruments LR50K est mise en œuvre à une vitesse traverse de $1 \mathrm{~mm} / \mathrm{min}$, une température d'essai de $23^{\circ} \mathrm{C}$ et une humidité de $50 \pm 10 \%$.

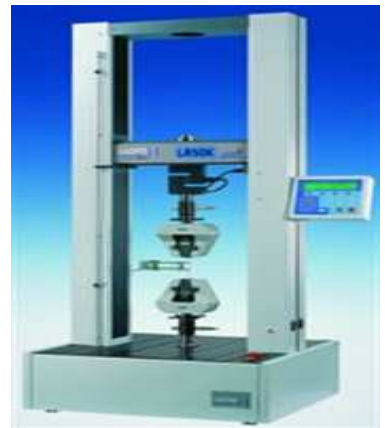

Fig.5. LLOYD Instruments LR50K

Le banc d'essai est équipé de mors auto-serrant et d'une cellule de force ayant une capacité de $5 \mathrm{kN}$. Son pilotage se fait par le logiciel Test expert qui permet à la fois de consigner les paramètres d'essais, d'acquérir et de traiter les données. 


\subsubsection{Résultats des essais pour le PVC dur vierge}

Table 1. Relevées enregistrées de l'essai de traction

\begin{tabular}{|c|c|c|}
\hline $\begin{array}{c}\mathbf{N}^{\circ} \\
\text { Eprouvette }\end{array}$ & $\begin{array}{c}\text { Contrainte à la } \\
\text { rupture (MPa) }\end{array}$ & $\begin{array}{c}\text { \% d'allongement } \\
\text { à la rupture }\end{array}$ \\
\hline $\mathbf{1}$ & 41,3 & 50 \\
\hline $\mathbf{2}$ & 40,0 & 60 \\
\hline $\mathbf{3}$ & 39,9 & 40 \\
\hline $\mathbf{4}$ & 38,1 & 50 \\
\hline $\mathbf{5}$ & 39,1 & 40 \\
\hline Moyenne & 39,7 & 48 \\
\hline Ecart-type & 1,05 & 7,48 \\
\hline
\end{tabular}

\subsubsection{Courbe de traction pour le PVC rigide vierge}

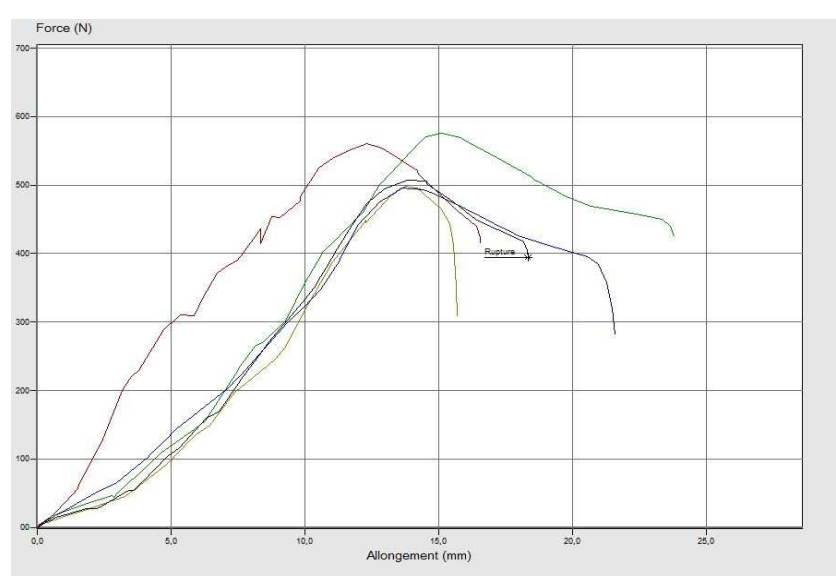

Fig.6. Courbes de traction

2.2.4 Résultats des essais pour le PVC rigide biochargé

Table 2. Relevées enregistrées de l'essai de traction

\begin{tabular}{|c|c|c|}
\hline $\begin{array}{c}\mathbf{N}^{\circ} \\
\text { Eprouvette }\end{array}$ & $\begin{array}{c}\text { Contrainte à la } \\
\text { rupture (MPa) }\end{array}$ & $\begin{array}{c}\text { \% d'allongement } \\
\text { à la rupture }\end{array}$ \\
\hline $\mathbf{1}$ & 23,7 & 25 \\
\hline $\mathbf{2}$ & 26,9 & 40 \\
\hline $\mathbf{3}$ & 38 & 25 \\
\hline $\mathbf{4}$ & 34,4 & 25 \\
\hline $\mathbf{5}$ & 33,6 & 30 \\
\hline Moyenne & 31,3 & 29 \\
\hline Ecart-type & 5,23 & 5,83 \\
\hline
\end{tabular}

\subsubsection{Courbe de traction pour le PVC rigide bio- chargé}

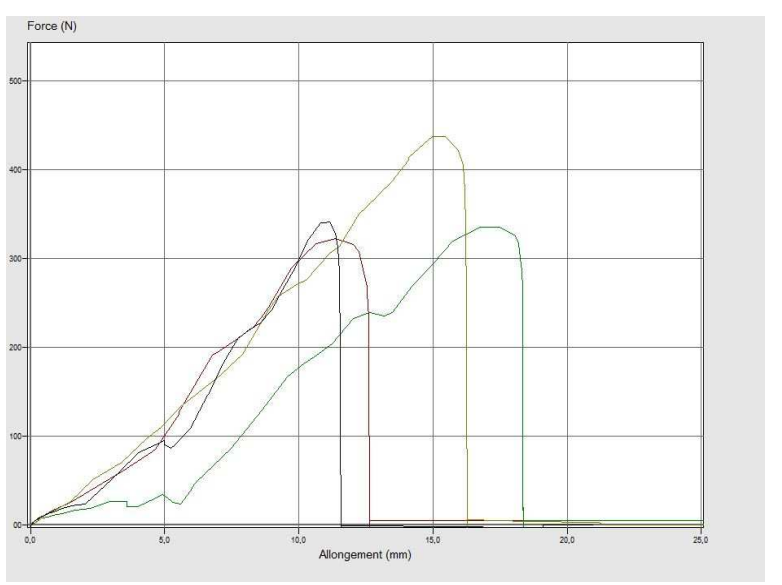

Fig.7. Courbes de traction

\subsection{Analyse du comportement élastique}

Nous pouvons observer les performances comportementales suivantes du nouveau éco-matériau face aux sollicitations d'extension :

La réactivité du PVC rigide aux sollicitations de traction a généré une variabilité de comportements élastiques intrinsèques, sachant que les comportements enregistrés relatifs aux cinq éprouvettes sont susceptibles de s'être imposés à force de leurs caractéristiques évaluées "Fig. 6 ", "Fig. 7 ".

$\mathrm{Au}$ cours de ces essais, les deux types de matériau ont subi plusieurs tests d'extension et on a retenu les valeurs expérimentales les plus significatives à savoir, la résistance maximale à la rupture et le déplacement relatif.

Nous pouvons observer, à travers les résultats des différents essais effectués sur les deux types des matériaux ainsi trouvés face à ces sollicitations que :

Les différentes courbes de traction obtenues présentent un comportement plastique avec seuil très marqué. Outre les paramètres de rupture, on définit une ligne parallèle à la tangente à l'origine des courbes à partir des différentes déformations pour marquer le coefficient directeur appelé module de Young ou module d'élasticité longitudinal caractérisant la dureté du matériau "Fig. 6", "Fig. 7 ".

Porté à une température de $185^{\circ} \mathrm{C}$, le biorenforcement à $10 \%$ de la charge en cause a contribué à une très sensible amélioration de la rigidité. Sur une même nuance, l'effet de durcissement est d'autant plus significatif que le niveau de dureté initial est faible et l'effet sur les caractéristiques de fragilité est d'autant moins appréciable que l'écart entre la dureté initiale et finale est élevé "Fig. 6", "Fig. 7".

Pour le lot d'éprouvettes du PVC basique, on remarque un comportement élastique non linéaire suivie d'une déformation plastique irréversible. Ce PVC basique subit une déformation plastique permanente avant de se rompre. C'est un matériau dur et ductile "Fig. 6 ". 
Pour le lot d'éprouvettes de l'éco-composite, l'étude a montré un comportement élastique, non linéaire et réversible. Apparition d'une zone semi-rectiligne, pour laquelle les allongements sont relativement proportionnels aux efforts appliqués. C'est la zone élastique qui est réversible, car si l'on supprime l'effort de traction, le retour à l'équilibre s'établit et l'éprouvette conserve un allongement rémanent. La rupture de l'écomatériau se produit alors qu'il est en régime de déformation purement semi élastique par rapport au cas initial. C'est un matériau rigide et fragile "Fig. 7".

Leger abaissement de la résistance à la traction sans aucune influence sur la valeur maximale à la rupture.

Importante chute d'allongement pour cent engendrant une amélioration de module de Young caractérisant une propriété de la matière de nouveau écocomposite accompagnée d'une diminution significative de ténacité "Fig. 7 ".

Variabilité de Comportements élastiques plus intense par rapport au PVC de base due au nouveau composant allié en substance organique qui a rendu le matériau final plus hétérogène. Cela a bien métamorphosé le comportement plus anistropique qu'auparavant "Fig. 6", "Fig. 7".

Disparition des zones de déformations plastiques du PVC basique après le bio-chargement "Fig. 6", "Fig. 7 ". Ce dernier procédé de transformation a permis finalement au matériau final de bénéficier de la propriété de dureté du matériau de base. En fait, la modification de la structure semi-cristalline du PVC bio-chargé est traduite par l'évolution du taux de cristallinité $《 x »$. Cet important paramètre se rencontre souvent dans le cas des matériaux organiques. Il mesure la proportion de matière se trouvant dans l'état cristallin et permet d'apprécier les contributions amorphes et cristallines. Une nouvelle structure qui se manifeste par une grande différence de densité entre phases amorphe et cristalline. Donc, la structure de la phase cristalline est devenue plus compacte [5].

En revanche, une amélioration de l'aspect de la morphologie du nouveau éco-composite à envisager par un ajustement de dosage et de température pour assurer une meilleure compatibilité du mélange. Cette supposition est très probable du fait que le taux de cristallinité massique $(x \mathrm{~m})$ ou le taux de cristallinité volumique $(x \mathrm{v})$, général peu différents, dépend des systèmes cristallins et de l'histoire thermique du polymère parent.

Aussi, il faudra disperser et distribuer les phases, souvent en présence de compatibilisants, ce qui nécessite généralement des études au cas par cas.

\section{Conclusion}

Actuellement, les axes de recherches sur Le développement des matériaux plus respectueux de l'environnement concernent bien les matériaux biosourcés, les matériaux biodégradables et les matériaux recyclés.

Dans cette perspective, nous avons pu, dans un premier temps, contribuer à la caractérisation élastique d'un matériau vierge d'origine animal. Ce test de résistance à la traction sur le banc d'essai dans des conditions similaires d'expérience, nous a permis de noter que ce comportement mécanique est jugé favorable à une mise en valeur de la bio-charge en cause pour l'innovation de certains matériaux polymères.

L'objectif recherché était d'analyser expérimentalement l'effet de cette biocharge sur la morphologie du PVC rigide.

A travers Les résultats expérimentalement obtenus, nous constatons que les hypothèses avancés par notre sujet de thèse sont vérifiés.

En termes de développement durable, deux approches principales seront développées dans cet axe de recherche : Le développement du recyclage des organes animaux en fin de vie grâce à l'amélioration d'identification des performances et le développement de matériaux bio-chargés de ressources renouvelables à partir de biomasses organiques.

\section{Obstacles Scientifiques}

Le développement de matériaux biosourcés et biodégradables nécessite l'évolution d'essais spécifiques d'évaluation de la biodégradation des matériaux formulés [10]. Ce développement de matériaux à partir de ressources première secondaires nécessite l'obtention de pureté de plus en plus importante afin de viser des applications à fortes valeurs ajoutées [11].

\section{Remerciements}

Nos remerciements vont au Laboratoire du Centre Technique de Plasturgie et de Caoutchouc (CTPC), accrédité NM ISO/CEI 17025, pour son accueil, les moyens mis à disposition pour mener à bonne fin cet article. Ce travail a notamment été rendu possible grâce à l'ensemble du personnel technique et administratif de ce laboratoire que nous tenons également à remercier.

\section{References}

1. E. Bretagne, J. Bréard, V. Massardier et V. Verney, "Eco-matériaux: Les matériaux passent au vert". 367-368, 100 (2012).

2. CA.Bernardo, AM.Cunha and MJ.Oliveira, the Department of Polymer Engineering, Universidade 
do Minho), "The Recycling of Thermoplastics: Prediction of the Properties of Mixtures of Virgin and Reprocessed Polyolefins", 4800 Guimaraes, Portugal, Polymer Engineering and Science, 36 (4) February (1996).

3. D.Dupoux, (Shell Chimie), "Economie-Ecologie du recyclage des matières plastiques", Colloque du Groupe Français D'Etudes et d'Applications des Polymères, Douai, 367-368 (1991).

4. A. Kouadri, S. Cretin, "Des évolutions indissociables ", (2007).

5. FS.Bates, "Polymer-Polymer phase behaviour", Science, 251 (4996), 898-905 (1991).

6. Y .Agari, M.Shimada. and A.Ueda, Polymer, 26492655, 38 (11) (1997).

7. M. Jammoukh, K. Mansouri et B. Sslhi, «Etude expérimentale du comportement élastique d'une biocharge de ressources renouvelables » RSE-2016. 6166, 1 (2016).

8. JC.Angola, Y.Fujita, T.Sakai, T.Inoue, "Compatibilizer-aided toughening in polymer.

9. L. AUGIER, "Etude de l'élaboration de matériaux composites PVC/bois à partir de déchets de menuiserie : formulation, caractérisation, durabilité et recyclabilité". 11-12, 2507 (2007).

10. Allred, Recycling process to scrap composites and prepregs, Int. SAMPE Symp.Exhib-1996. 61-66, 41 (1996).

11. K. Halada, "Progress of ecomaterials toward a sustainable society, Current Opinion in Solid State and Materials Science". 209-216-368, 7 (2003). 